\section{Active Database Rulebase}

AnnMarie Ericsson, Mikael Berndtsson, and Jonas Mellin

University of Skövde, Skövde, Sweden

\section{Definition}

An active database rulebase is a set of ECA rules that can be manipulated by an active database.

\section{Key Points}

An active database rulebase is a set of ECA rules that can be manipulated by an active database. Thus, an ADB rulebase is not static, but it evolves over time. Typically, ECA rules can be added, deleted, modified, enabled, and disabled. Each update of the ADB rulebase can potentially lead to different behaviors of the ECA rules at run time, in particular with respect to termination and confluence.
Termination concerns whether a set of rules is guaranteed to terminate. A set of rules may have a nonterminating behavior if rules are triggering each other in a circular order, for example, if the execution of rule $\mathrm{R} 1$ triggers rule $\mathrm{R} 2$ and the execution of rule R2 triggers rule R1. A set of rules is confluent if the outcome of simultaneously triggered rules is unique and independent of execution order.

\section{Cross-Reference}

- ECA Rules 